\title{
ELEMENTAL QUANTIFICATION OF HAFNIUM-SILICATES BY ELECTRON ENERGY LOSS SPECTROSCOPY
}

\author{
Mark Clark ${ }^{*}$, Brendan Foran ${ }^{*}$, Carolyn Gondran ${ }^{*} \&$ Patrick S. Lysaght ${ }^{* *}$ \\ *ATDF, 2706 Montopolis Dr. Austin, TX 78741 \\ ***SEMATECH, 2706 Montopolis Dr. Austin, TX 78741 \\ email: Mark.Clark@ATDF.com
}

Hafnium-silicate thin films are potential candidates to replace $\mathrm{SiO}_{2}$ as the gatedielectric in semiconductor devices [1]. Elemental analysis of the gate-dielectric composition is necessary to understand relationships between processing conditions and electrical performance. Electron energy loss spectroscopy (EELS) recorded in a scanning transmission electron microscope (STEM) has been used for spatially resolved quantification of traditional gate dielectric stacks [2]. However, previous characterization of hafnium-silicates has been limited to qualitative elemental analysis [3], in part due to complications from plural scattering. In this work the composition of hafnium-silicate films is quantified using Hartree-Slater partial ionization crosssections.

EEL spectra were recorded for hafnium-silicate films, as deposited by atomic layer deposition (ALD), with concentrations ranging from 30 to $63 \mathrm{~mol} \% \mathrm{SiO}_{2}$ as verified by Rutherford backscatter spectrometry (RBS), (Table 1). TEM cross-sections were prepared by focused ion beam (FIB) [4], as this technique provided a more planar surface than ion milling, which tended to make the hafnium-silicate layer bulbous due to differential milling (Figure 1). Both high and low energy-loss spectra were acquired at $1 \mathrm{eV} /$ pixel and stitched together (Figure 2). Elemental quantification was performed using commercially available software [5] to analyze the O-K, Hf- $\mathrm{M}_{4,5}$ and $\mathrm{Si}-\mathrm{K}$. Sample thicknesses less than $0.3 \mathrm{t} / \lambda_{\mathrm{Si}}(<60 \mathrm{~nm})$ were necessary to minimize plural scattering effects that otherwise hindered accurate quantification [6].

Elemental compositions of ALD hafnium-silicate films quantified by comparing coreloss intensities using Hartree-Slater determined partial ionization cross-sections are in good agreement with RBS data (Table 1). However, the success of this approach required long acquisition times of the low intensity $\mathrm{Hf}_{-} \mathrm{M}_{4,5}$ and $\mathrm{Si}-\mathrm{K}$ signals, while rastering a small probe over a large area to prevent beam-induced sample damage. For dielectric thin film process development, it would be desirable to measure changes in stoichiometry across a 3-4 nm thick dielectric film with sub-nanometer spatial resolution. To this end, multiple least squares (MLS) fitting of low-loss spectra, acquired with low probe current and short spectral acquisition time, is used for quantification at high spatial resolution.

[1] G. D. Wilk, R. M. Wallace, and J. M. Anthony, J. Appl. Phys. 89, 5243 (2001).

[2] H. Stegmann and E. Zschech, Appl. Phys. Lett. 83, 5017 (2003)

[3] B. Foran, J. Barnett, P. S. Lysaght, M. P. Agustin and S. Stemmer, J. of Electron Spectroscopy and Related Phenomena 143, 151 (2005). 
[4] Sample preparation was performed by Maria Hall and Charlene Johnson at ATDF.

[5] Digital Micrograph V. 3.7.1. Gatan Inc. http://www.gatan.com

[6] N. Zaluzec, $41^{\text {st }}$ Ann. Proc. Electron Microsc. Soc. Am., 388 (1983).

TABLE 1. ALD hafnium-silicate film compositions as quantified by RBS and EELS.

\begin{tabular}{c|ccc|ccc} 
& \multicolumn{3}{|c|}{ RES (at.\%) } & \multicolumn{3}{c}{ EEIS (at.\%) } \\
mol.\% SiO $\mathbf{2}_{\mathbf{2}}$ & Hf & $\mathrm{Si}$ & O & Hf & Si & O \\
\hline 30 & 22 & 10 & 68 & 22 & 9 & 67 \\
45 & 18 & 15 & 67 & 17 & 16 & 67 \\
54 & 15 & 18 & 67 & 14 & 19 & 67 \\
63 & 11 & 21 & 68 & 10 & 22 & 68 \\
\hline Uncertainty & 1 & 1 & 4 & 1 & 1 & 3
\end{tabular}
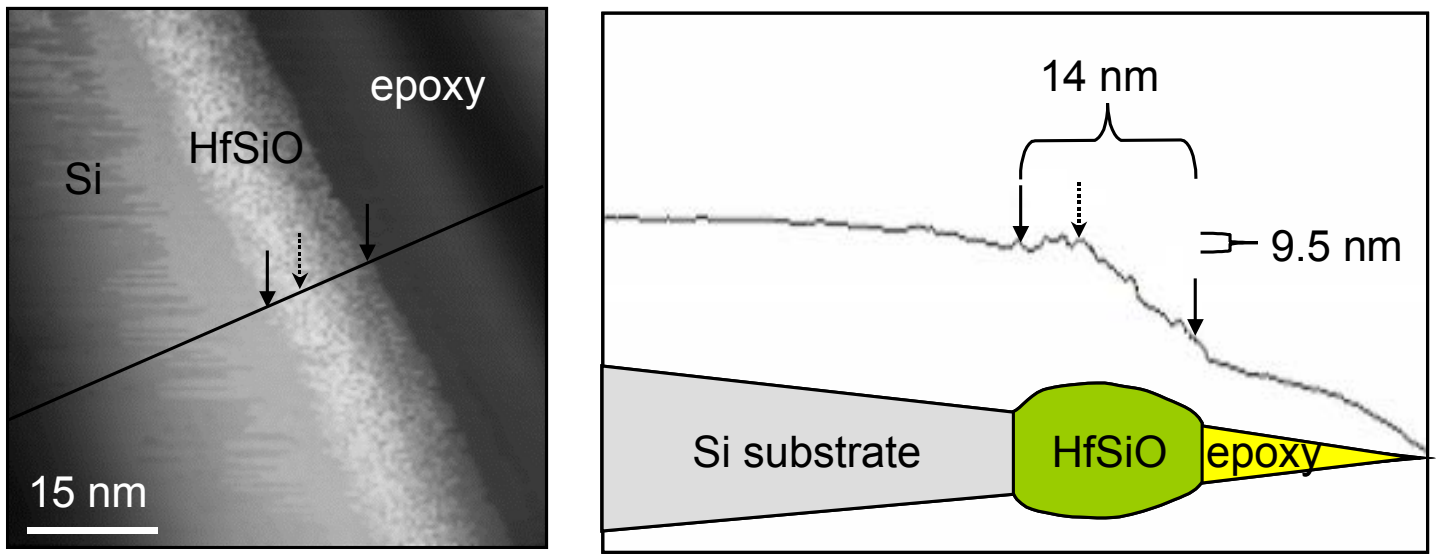

Fig 1. Atomic force microscopy (AFM) image of a 63 mol. $\% \mathrm{SiO}_{2}$ sample prepared in cross-section by ion milling and corresponding surface profile and cartoon.

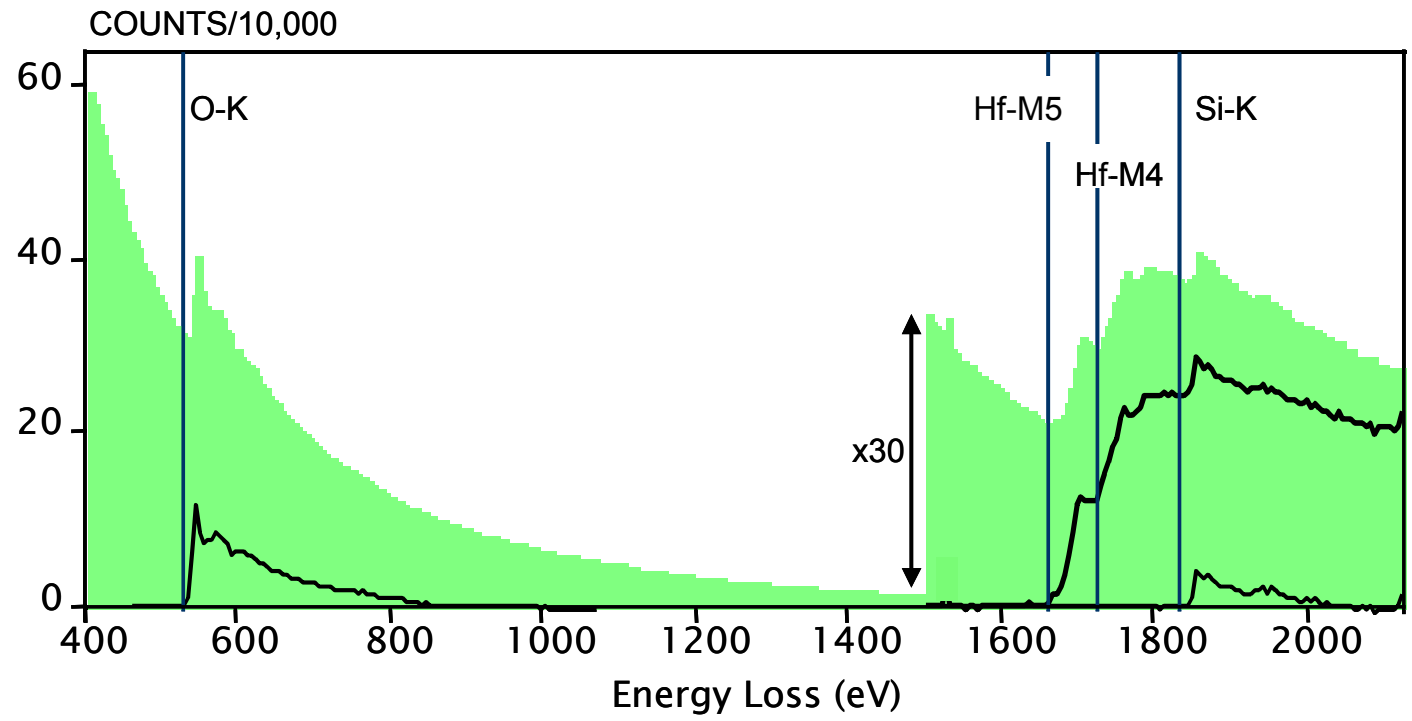

Fig 2. Electron energy loss spectra from a $40 \mathrm{~mol} \% \mathrm{SiO}_{2} \mathrm{ALD}$ hafnium silicate were acquired at a dispersion of $1.0 \mathrm{eV} / \mathrm{pixel}$. The integration windows were from 640 to 670 eV for O-K, 1790 to $1820 \mathrm{eV}$ for Hf-M4,5 and 1899 to 1929 for Si-K. 